\title{
Frecuencia del Macroorquidismo en Pacientes con Retraso Mental: Según el Criterio de Evaluación
}

\author{
Dr. Alejandro Zavala B.; Dr. Jves Lacassie S. 1. \\ Frecuency of Macroorchidism in Mentally \\ Retarded Patients: According to \\ Evaluation Criteriae
}

\begin{abstract}
On the phenotypic screening of the Fragile-X Syndrorne in an institution for the mentally retarded (MR), a high frequency of macroorchidism (MO) was found using international values for testicular volumes (TV) 1 . The report of normal TV in chilean school-age children ${ }^{2}$, led us to reevaluate the prevalence of MO using these values. As these are slightly higher than foreign measurements, the percentage of $\mathrm{MO}$ found in the older group ( $>15$ y.0) decreased from 28.57 to 23.9 ( $>>0.50$ ). However the total frequency of macroorchidics changed from 17.64 to 18.05 $(p>0,50 \%)$ due to a relative increase $(6.97$ to $10 \%)$ of $\mathrm{MO}$ in the youngest group. This incrament was not due to the subtle differences between the reference values but to the actual cxclusion of $27.9 \%$ of $M R$ subjects with bilateral cryptorchidism (14.11\% in the total group). The prevalence of MO in the older group, according to other standards used in similar studjes, showed differences ranging from $28.57 \%$ to $12.82 \%$ According to our criteriae of evaluation the use of normal chilean vaiues for the determination of $\mathrm{MO}$ in our country seems to be convenien1. The methods for the clinical evaluation of MO are brietly reviewcd

(Key words: Macroorchidism: Mental retardation. Testicular volumc).
\end{abstract}

La delineación del Síndrome del X-frágil en los últimos años, su reconocimiento como una causa frecuente de retraso mental en todos los países donde se te ha investigado y la aparente respuesta al tratamiento con ácido fólico han despertado gran interés en él. ${ }^{3-10}$. Una de las características clínicas que más ha llamado la atención y permitido su búsqueda fenotípica es la presencia de macroorquidismo (MD) ${ }^{1} \cdot 3 \cdot 11$ 12 , evidente no sólo en la pubertad, sino en algunos casos en la niñez, en el recién nacido e incluso en el feto ${ }^{3}-13-16$. En la búsqueda de este síndrome en pacientes deficientes mentales institucionalizados, basada en la presencia de $\mathrm{MO}^{1}$, fue necesario evaluar el volumen testicular (VT) y definir MO utilizando tablas extranjeras $^{17}$. La publicación de VT normales para la población escolar chilena por Valenzuela y col en $1983^{2}$, nos ha motivado a reevaluar la población

1. Unidad de Genética, Instituto de Nutrición y Tecnología de los Alimentos (INTA), Universidad de Chile. Santiago-Chile.

Trabajo presentado en las X Jornadas Nacionales de Pediatría Viña del Mar, 1983, y Financiado por DIB. U. de Chile.

El Dr. Zavala, en la actualidad es Becado en Cirugía Infantil, Hospital Dr. E. González Cortés. estudiada, usando estos valores y revisar las diferentes técnicas de medición testicular, discutiendo algunos de los problemas que su uso conlleva.

La medición del VT constituye sólo una apro. ximacion. Numerosas técnicas y cifras de VT "normales" han sido publicadas. Una enumera. ción cronológica de algunas de ellas se presenta en la Tabla 1. Las diferencias de VT en algunas poblaciones han sido atribuidas fundamentalmente a diferencias étnicas ${ }^{18} \cdot 19$. En los asiáticos se han descrito VT menores que en cauca sicos y negros, entre los cuales no habría di . ferencias ${ }^{1} 1-19$. Las etapas de maduración testicular serían las mismas en diferentes poblaciones pero con variaciones en el tiempo y tamaño alcanzado ${ }^{20}$. Aunque hay correlación entre VT y edad ósea, así como entre VT y edad cronológica, la mejor correlación del VT sería con la etapa de desarrollo pubera! 1 7-19.

La medición del.VT se realiza principalmente a través de modelos comparativos (orquidómetro) y por fórmulas matematicas. En la actualidad se están utilizando plantillas volumétricas con volúmenes estandarizados en una población brasileña ${ }^{21}$. Los orquidómetros son collares con elipses de volúmenes determinados. El más utilizado es el orquidómetro de $\operatorname{Prader}^{17} \cdot 22-23$, aunque también existen otros modelos $24-25$. 
Tablin 1 .

Volumen testicular. Síntexis cronológica de valores publicados

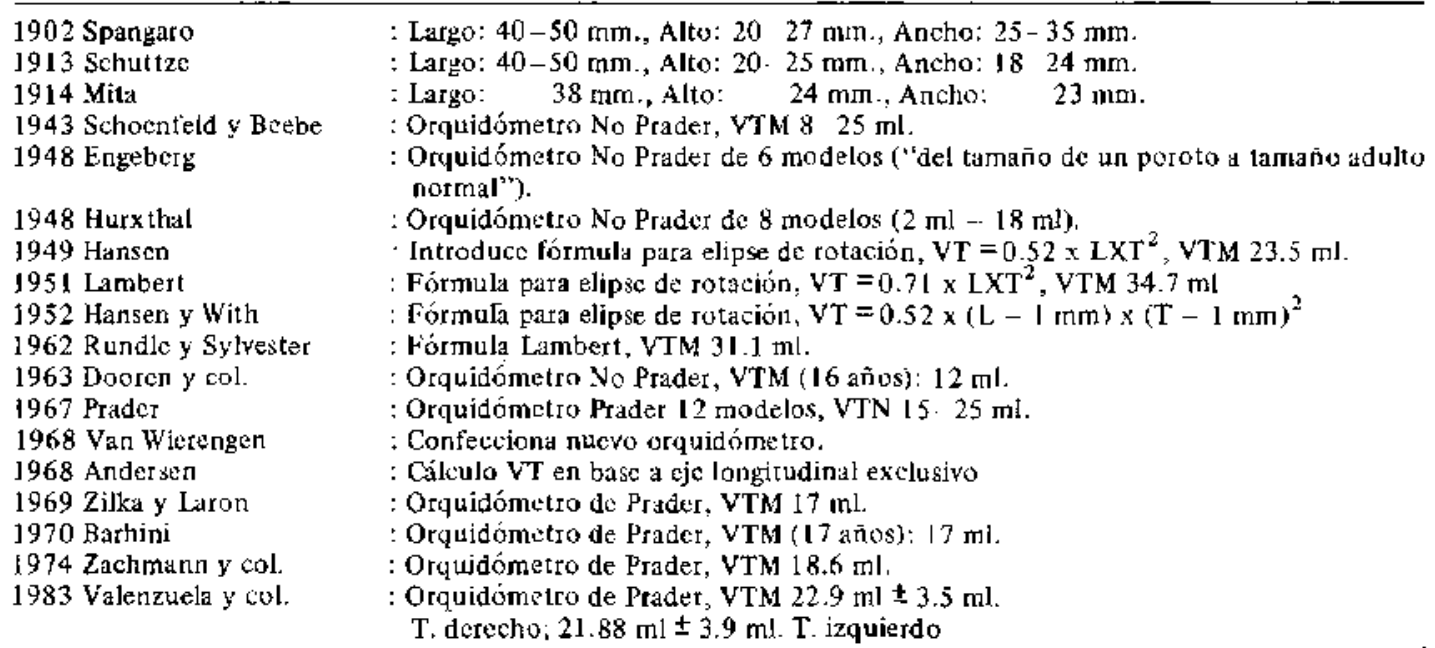

VT $=$ Volumen Testicular

VTM $=$ Volumen Testicular Medio.

Los orquidómetros tienen la ventaja de ser de fácil manejo, con escasa manipulación genital, y, en el caso deI oreuidómetro de Prader, de haber sido utilizado en diferentes poblaciones 17-22 Tienen la desventaja de ser un método comparati. wo, por lo que se requiere entrenamiento para su uso adecuado, y tener volúmenes fijos, por to que volúmenes intermedios son determinados splamente por aproximación ${ }^{2}$. Ademas no permiten la cuantificación det VT mayores como sucede con los casos de macroorquidismo que nos intere. san.

Las fórmulas matemáticas requieren medir los ejes longitudinal y transversal, utilizando una regla métrica de plástico o calibrador micrométrico (cảliper). La más utilizada es la fórmula de Hansen (VT $=0.52 \times \mathrm{L} \mathrm{x} \mathrm{T}^{2}$ ) $^{23}$, introducida al estudio del X-frágil por Cantú y $\operatorname{col}^{13}$. Las fórmulas tienen la ventaja de requerir sólo acu. ciosidad en la medición y no el mayor entrena. mjento necesario para comparar con los orquidó. metros. Además el instrumento que se requiere. la regla métrica, está al alcance de cualquier médico. Tiene la desventaja de requerir mayor manipulación genital y la técnica de medición no estar totalmente estandarizada, pudiendo existir diferencias en la determinación de los ejes (por ejemplo, si se incluye o no en la valoración el epidídimo o el espesor del escroto) con las consecuentes variaciones de] VT.

Utilizando estos métodos se han publicado diferentes tablas obtenidas en diversas poblaciones, habitualmente en estudios transversales, con distintos rangos de "normalidad" (Fig. 1). Algu- nas de éstas son criticables, como es el caso de la curva A obtenida en una institucion para deficientes mentales ${ }^{25}$. La posible inclusión de sujetos con el sindrome del $X$-frágil o el sindrome de $\mathrm{RM}+\mathrm{MO} \sin$ fragilidad cromosómica ${ }^{26-27}$, frecuentes en estas instituciones $^{3}$, pudiera explicar el mayor VT encontrado.

\section{MATERIAL Y METODO}

En el trabajo preliminar se calcularon los VT de 85 sujetos con RM internados en el Pequeño Cottolengo (Santiago, Chile), utilizando la fórmula de Hansen ${ }^{1}$. Se definió como macroorquidico al sujeto con VT mayor a +2 DS para la edad, de acuerdo a las tablas de Zachmann ${ }^{17}$. En estas tablas el VT medio entre los 12,5 y los 19.20 años fue obtenido en un estudio transversal, y los valores entre los 10 y 12 años en un estudio longitudinal. En el trabajo previo se consideró e] VT mayor en forma independiente, y en los menores de 10 años, no considerados en la tabla de Zaclumann. se utilizó como valor "normal", en forma arbitrarja pero más exigente, e] volumen de los 10 aftos.

En el presente trabajo se compara la distribución de los VT obtenidos en el primer estudio, con la obtenida por las tablas de Valenzuela y col y con la de Zacliniann, considerando sólo los VT medios cuando fue posible y sólo para mayores de 10 años. Además se compara la variación en la incidencia de $\mathrm{MO}$ de acuerdo al grado de desarrollo puberal de Tanner para VT medio y al criterio de Howard-Peebles en los mayores de 15 años. 
criterios que son utilizados en algunos trabajos.

En la Tabla 2 se presenta el número de sujetos en que no había testículos palpables o sólo uno de ellos estaba descendido, lo que impide en estos casos calcular el VT medio. El chi cuadrado considerando los normales criptoquídicos uni y bilateral de acuerdo a la edad, es altamente significativo $\left(\mathrm{X}^{2}=16.0296: \mathrm{p}<0.001\right)$.

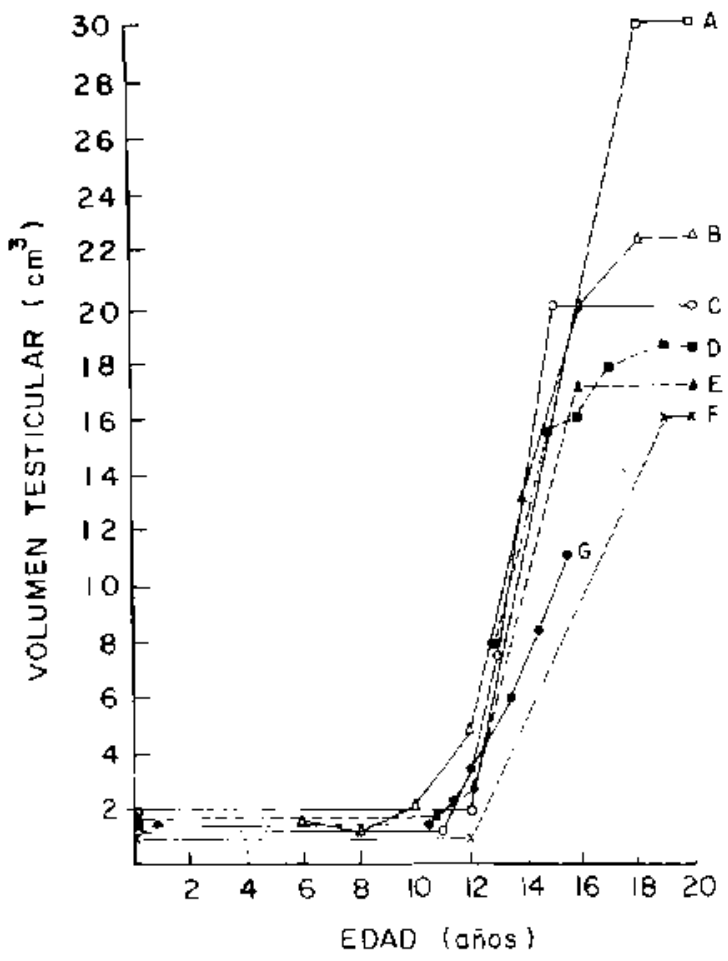

Figura 1. A) Rundle y Sylvester: Arch. Dis. Child. 3? 514, 1962 (Formulis Lambert. Pobl. de RM Caterham); B) Valenzuedi et al.; Rev. Chil. Pedialr. 54: 240, 1983 (O. de Prader. Pobl. liscolar Santiago Norte); C) Prader:: Triangle 7: 240, 1966 (O. de Prader - Pobl $b$; D) Zachinann y Prader.: Helv. Pacdiat. Acta 29: 61, 1974. (O. de Prader. Pobl. Zurich); 1) Zilka y Laron.: J. Clir. Endoctinol. Mctab. 29: 1409. 1969. formula Hansen y With. Potl. Istaci). I) Schoenfeld y Beebe: J. Urol. 48: 759, 1942. (O. no Prader Pobl.) (j) Dooren et al.: Med. T. Gencesk. I07: 1519, 1963. (O. no Prader Publ. Holandesi).

Adaptado de Laron, Z. y Zilka: E.J. Clin. Findocrinol Metab. 29: $1409,1969$.

Tabla 2 .

Frecuencia clínica de testiculos no descendidos

\begin{tabular}{lccrccc} 
& \multicolumn{3}{c}{$\begin{array}{c}\text { Sólo un testículo } \\
\text { palpable }\end{array}$} & \multicolumn{2}{c}{$\begin{array}{c}\text { Sin testiculos } \\
\text { palpables }\end{array}$} \\
\hline Fdad (atoos) & $\mathrm{N}$ & $\mathrm{N}$ & $\%$ & $\mathrm{~N}$ & $\%$ \\
\hline$<15$ & & 43 & 6 & 13.95 & 12 & 27.9 \\
$>15$ & 42 & 3 & 7.14 & & \\
\hline TOTAL & 85 & 9 & 10.58 & 12 & 14.11 \\
\hline
\end{tabular}

\section{RESULTADOS}

En la Tabla 3 se presenta el porcentaje de MO en la población total y separada en mayores y menores de 15 años, para cada uno de los criterios de evaluación utilizados. Los resultados obtenidos con las tablas de Valenzuela (columna C) no demuestran diferencias significativas (p $>0.05)$ al compararlos separados por grupos etarios, con los obtenidos por Zachmann (columnas A y B) utilizando la prueba de chi cuadrado, la de Fischer o ambas.

En la Figura 2 se observa la distribución del VT medio para la edad de cada uno de los sujetos en las curvas de VT medio de Zachmann y col. (A), Valenzuela y col. (B) y según el ctiterio de Tanner (C).

\section{DISCUSION}

Dado que no existen diferencias significativas entre los VT obtenidos con orquidómetro de Prader y la fórmula de Hansen ${ }^{19 \cdot 22}$, por facilidad práctica $y$ por el hecho de que al estudiar pacientes con MO frecuentemente se encuentran valores fuera del rango del orquidómetro, hemos utilizado y recomendamos medir el largo $y$ ancho testicular con regla métrica $y$ calcular el VT mediante la fórmula de Hansen. $\Lambda$ ntes de nuestro trabajo preliminar, en Chile se habian publicado sólo tablas para VT derecho ${ }^{28}$. La publicación de valores para ambos testiculos permite tanto la evaluación de MO unilateral como el uso de valores promedio ${ }^{2}$, Varios trabajos $26 \cdot 29$ han utilizado el percentil 90 de VT (23 $25 \mathrm{ml}$ ) como limite superior normal. Nosotros hemos considerado MO a todo sujeto con volútnenes mayores a +2 DS para la edad correspondiente. El alto número de sujetos retrasados mentales sin testiculos palpables, especialmente entre los menores de 15 años (Tabla 2) hizo que en el trabajo preliminar fueran considerados dentro del grupo "normal" o no macroorquídico, to que implicaría una eventual subestimación de la población con MO. En este trabajo se excluyeron los sujetos sin testículos palpables o aquellos con sólo uno cuando la tabla de comparación utilizaba VT medio, incluyéndose en la categoría No Aplicable (NA). La gran proporción de criptorquídea encontrada ha side también descrita, recientemente, en poblaciones de deficientes mentales en EE.UU, ${ }^{30}$

Los 12 sujetos con MO detectados inicialmente en el grupo mayor de 15 años disminuyen a 10 al aplicar la tabla chilena, debido a que sus valores son más exigentes. En otras palabras, la población chilena estudiada tiene volúmenes testiculares algo mayores que poblaciones caucásicas 
extranjeras (Fig. 2), En los menores de 15 años se mantuvo el número de sujetos con M.O. Al excluir sin considerar como "normales a los 12 sujetos sin testículos palpables y a un menor de 6 años, el porcentaje de $\mathrm{MO}$ aumentaría de 6.97 a $10 \%$ (Tabla 3.c). Esto sucede porque el número de sujetos disminuye de 43 a 30 , manteniéndose Jos 3 sujetos con MO. La utilización de las Tablas de Zachmann considerando VT medio disminuye el número de $\mathrm{MO}$ de 3 a 1 , indicando que sólo existía MO unilateral. Sin embargo, el mayor número de sujetos con testículos altos uni o bilateral reduce la población aplicable a 20 (columna B) disminuyendo el porcentaje de $\mathrm{MO}$ a $5 \%$. Esto demuestra la conveniencia de utilizar la tabla chilena con valores individuales en nuestra población. La mayor proporción de M.O. que resulta con la tabla chilena en comparación con la de Zachmann medio (columna $C$ vs B), tam- bién se observa en los mayores de 15 años. Si bien el porcentaje de MO en la población estudiada sería levemente inferior al utilizar las tablas chilenas con las mismas condiciones o criterios, el porcentaje de positividad se mantiene cuando ambas tablits son correctamente aplicadas. Con las tablas de Tanner y Howard-Peebles se obtuyieron los valores más bajos de MO. Estas utilizan VT promedios, su uso restringido en la niñez y lo arbitrario de la última hacen que sean menos utilizadas en la práctica clínica.

Dado que pueden haber diferencias étnicas ${ }^{2}$. $11-18-19$ y de hecho las mediciones de la población chitena son mayores $y$ con mayor dispersión (Fig. 2), parece recomendable el uso de las Tablas de Valenzuela y col. en nuestro medio. Para edades inferiores a 6 años, mientras no existan estudios nacionales, parece adecuado usar la Tabla de Prader $^{23}$, y en los menores de 1

Tabla 3.

Frecuencia de matroorquidismo de acuerdo al ariterio de evaluación.

\begin{tabular}{|c|c|c|c|c|c|}
\hline $\begin{array}{l}\text { Tablas } \\
\text { VT usado }\end{array}$ & $\begin{array}{c}\text { A } \\
\text { Zachmann }^{12} \\
\text { Indep. }^{1}\end{array}$ & $\begin{array}{c}\text { B } \\
\text { Zachmann } 2 \\
\text { Promedio }\end{array}$ & $\begin{array}{c}c \\
\text { Valenzucla } \\
\text { Indep. }\end{array}$ & $\begin{array}{c}\text { D } \\
\text { Tanner }^{12} \\
\text { Promedio }\end{array}$ & $\begin{array}{c}\mathrm{li}^{\mathrm{c}} \\
\text { Howard-Peebles } \\
\text { Promedio }\end{array}$ \\
\hline \multicolumn{6}{|l|}{$\begin{array}{l}\text { Mayores de } \\
15 \text { ation }\end{array}$} \\
\hline $\mathrm{N}$ & 42 & 39 & 42 & 39 & 39 \\
\hline N... ${ }_{-}^{d}$ & 0 & $3^{a}$ & 0 & $3^{2}$ & $3^{a}$ \\
\hline Normales & 30 & 31 & 32 & 32 & 34 \\
\hline м.o. & 12 & 8 & 10 & 7 & 5 \\
\hline$\%$ M.O. & 28.57 & 20.51 & 23.8 & 17.94 & 12.82 \\
\hline $\begin{array}{r}\text { Menores de } \\
15 \text { ̄̃os } \\
\\
\mathrm{N}\end{array}$ & 43 & 20 & 30 & 24 & \\
\hline N.A. ${ }^{d}$ & 0 & $23^{a, b}$ & $13^{a, b}$ & $19^{a, b}$ & \\
\hline Normales & 40 & 19 & 27 & 24 & \\
\hline M.O. & 3 & 1 & 3 & 0 & \\
\hline$\%$ M.O. & 6.97 & 5.00 & 10 & 0 & \\
\hline Total Pob. Aplic. & 85 & 59 & 72 & 63 & \\
\hline \%.O. & 17.64 & 15.25 & 18.05 & 11.11 & \\
\hline
\end{tabular}

${ }^{a}$ No se puede calcular VTM

${ }^{b}$ Quedan fuera de la Tabla por edad

$\mathrm{c}_{\mathrm{MO}}=\mathrm{VT}>34 \mathrm{ml}$

d No aplicable 


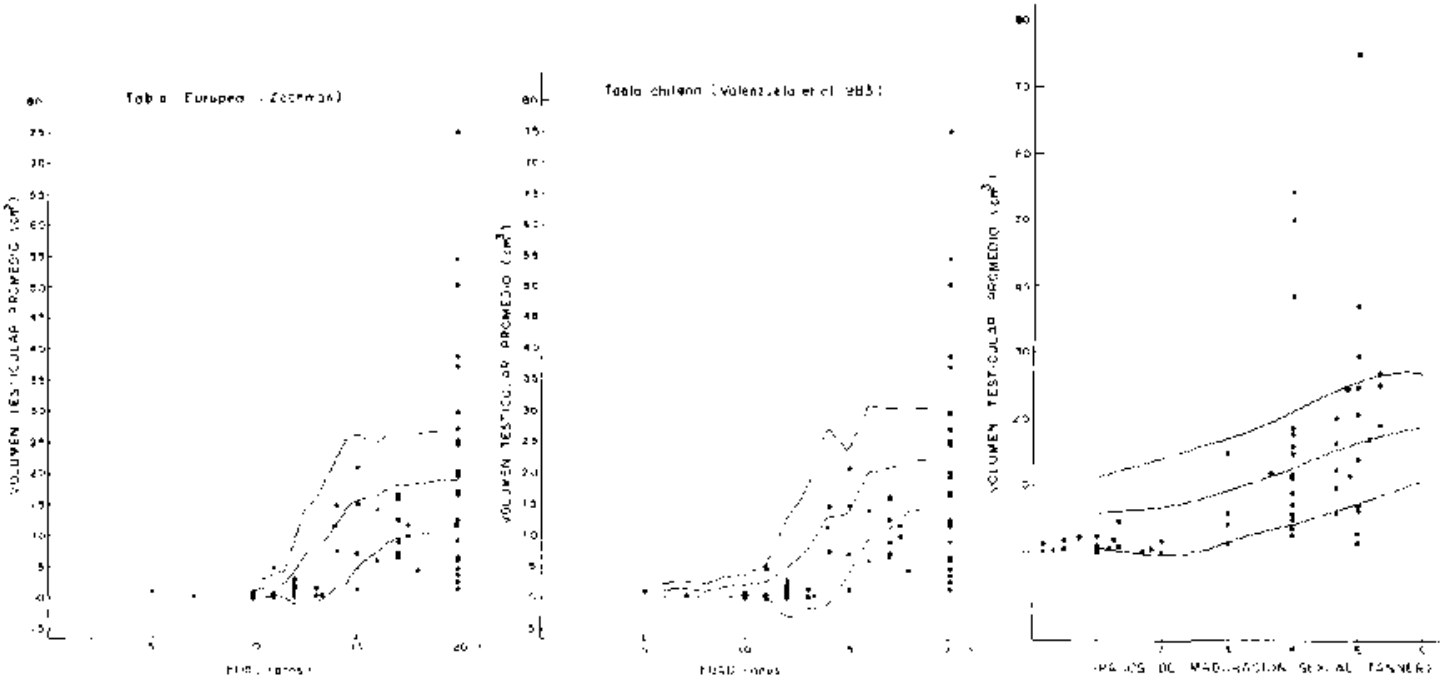

Figura 2.: Distribución del polumen testicular medio adaptado de Zachmann y col. (A), Valenzuela y col. (B), Tanner (C)

año, la Tabla de Cassorla y col. ${ }^{24}$ que seria la única con VT medidos en forma mensual.

En la evaluación de pacientes con RM, con o $\sin$ antecedentes familiares, debe investigarse el MO por la posibilidad de detectar un síndrome del X-frágil o de Martín Bell ${ }^{3}$. La descripción de MO aún en etapa fetal en este sindrome y la alta frecuencia de criptorquidea entre deficientes montales bacen plantear la posibilidad de que al MO pudiese constiluir una causa de criptorquidea, haciendo interesante investigar este sindromc en pacientes con criptorquidea uni o bilateral y RM o retardo del desarrollo psicomotor.

\section{RESUMEN}

En el rastreo fenotípico del síndrome del $\mathrm{X}$-frágil en una institución para retrasados mentales a través de la medición del volumen testicular (VT), se encontró una alta frecuencia de macroorquidismo (MO) ${ }^{1}$. En ese estudio se utilizaron tablas extranjeras de VT. La publicación de VT normales en una población escolar chilena ${ }^{2}$ nos motivó a reevaluar la prevalencia de $M O$ usando estos valores que son ligeramente mayores. En la población mayor de 15 años, el porcentaje de MO disninuyó de 28,57 a $23,9 \%$ ( $\mathrm{P} 0.50$ ). Sin embargo, la frecuencia total de MO aumentó de 17,64 a $18,05 \%$ (p 0.50 ) debido a un aumento relativo de menores de 15 años con $\mathrm{MO}(6,97$ a $10 \%)$ como consecuencia de la exclusión de un $27,9 \%$ de niños con criptor. quidea bilateral. Esta estaba presente en el $14,11 \%$ del total de la población estudiada. A! utilizar otros criterios empleados en estudios similares, la prevalencia de MO en el grupo post -..puberal varió entre 12,82 y $28.57 \%$.

De acuerdo a nuestro criterio de evaluación, parece altamente recomendable el uso de los valores normales chilenos para determinar $\mathrm{MO}$ en nuestro país.

Se revisan además los métodos de evaluación de VT utilizados en clínica.

\section{REFERENCIAS}

1. Lacassie, Y., Lavala, A., Curoito, B., Aliende, M.A., De Andraca, l.: Búsqueda fenotípica del RM ligado al sexo: Sindrome del Xq fra. Rev. Chil. Pediatr. 53: 419,1982 .

2. Valenzuela, C., Avendaño, A., Patri, A., Seprilvecia, $H$.: Tamaño testicular en escolares. Rev. Chll. Pediatr. 54: 240, 1983

3. Opirz, I.M., Sutherland, G,R.: Conference Report: International Workshop on the fragile $X$ and $X$-linked mental retardation, Am. J. Med. Genct. $17: 5,1984$

4. Rhoades, F.A.: X-linked mental retardation and fragle $X$ or marker X Syndrome. Pediatrics. 69. 668,1982 .

5. Lejeune, $J_{\text {.: }}$ Is the fragile $\mathrm{X}$ syndrome amenable to t'ratment? Lancet 1: 273, 1982.

6. Harpey, J.P.: Treatment of tragile-X. Pediatrics 69 : 670,1982 .

7. Brown, W.T., Jenkins, E.C., Friedman, E., Brooks, J., Cohen, J.L., Duncan, L., Hill, A.L., Matik, M.N., Morris, V., Wolf, E. W'isniewski, K., French, J.H.: Folic acid therapy in the fragile $X$ syndrome. Am. J. Med. Genet. 17: 289, 1984

8. Carpenter, N.J., Barber, D.H., Janes, M., lindley, W. Carr, C.: Controlled six month study of oral folic acid therapy in boys with Fragile $\mathrm{X}$-linked mental retardation. Am. T. Hum. Genet. 35: 82A, 1983.

9. Hagerman, $R$, McBogg, P., Levitus, A, McGavron, L., Smith, A., Berry, B., Braden, M., Van Housen. $K$. Newati, $K$., Matus, $I$.: Folic acid treatment of 
the Fragile X Syndrome. Atm. J. Hum. Gener. 35: $92 A, 1983$.

10. Lacassie, Y., Curotto, B., Alliende, M.A., De Andraca, $L$, Zavala, A.: livaluación preliminar del tratamiento con ácido fólico en dos pacietstes con retreso mental ligado al sexo y macroorquidismo. Rev. Med. Chil. 112:469, 1984 .

11. Rhoades, $F$.A.: Fragile X-syndrome in Hawaii: A summary of clinical experience. Am. J. Med. Genet. 17; $209,1984$.

12. Brown, W.T., Mezzappa, P.M., Jenkins, E. C: Screening for Fragile $X$ syndrome by testicular size measurement. Latncet 2: 1055, 1981.

13. Cantu, J.M., Scoglia, H.E., Medina, M., GonzálezDidde, M., Morato, T, Moreno, M.E., Pérez-Palacios. Inherited congenital normofunctional testicular hyperplasia and mental deficiency. Hum. Genet. $33: 23,1976$.

14. Jenkins, E.C., Brown, W.T., Brooks, J., Duncan, L.J., Rudelit, R.D., Wisniewski, H.M.: Fxperience with prenatal fragile $\mathrm{X}$ detection. Am. $\mathrm{J}$. Med. Genct. 17: 215, 1984.

15. Corpenter, N.J., Leichtman, L.G., Say, B.: Fragile $\mathrm{X}$-linked mental retardation (A survey of 6.5 patients with Mental Retardation of Lnk1iown origin). Am. J. Dis. Child. 136: 392, 1982.

16. Rudelli, R.D., Jenkins, E.C., Wisniewski, K, Moretz, R., Byrne, J., Brown, W.T.: Testucular size in fetal fragile $X$ syndrome. Lances 1: $1221,1983$.

17. Zachmann. M. Prader, A., Kind, H.P., Hofliger, H., Budliger, $H .:$ Testicular volume during adolescence. Hclv. Paediat. Act. 29:61, 1974.

18. Schonfeld, W.A., Becbe, G.W.: Normal growtl and variation in male genitalia from birth to maturity. $J$. Urol. 48: 759, 1942 .

19. Dantel, W.A., Feinstein, R.A., Howard-Peebles, P.N., Baxley, W.D.: Testicular volume of adoles- cence. J. Pediatr. 101: 1010, 1982.

20. Gardner, L,L:- Fndocrine and genetic diseases of thildhood and adolescence. W.B. Saunders Co., Philadelphia. Segundi Edición, 1975.

21. Bustos, E.: Comunicación personal, 1983.

22. Laron, Z., Tilka, E.: Compensatory hypertrophy of testicle in urilateral cryptorchidisin. J. Clin. I:ndocrinol, Metab. 29: 1409, 1969

23. Proder, $A$.: E] tamano de los testículos. Valoración. y signiticación clínica. Triángulo 7: 240, 1967.

24. Cossoria, F. G., Golden, S.M., Johnsenbaugh, R.A., Heroman, W.M. Loriatix, D.L., Sherins, R.J.: Testicular volume during carly infancy. J. Pediatr. 99: $742,1981$.

25. Rundle, A.J., Sylvester, P.E.: Measutement of testicular volume. Arch. Dis. Child. 37: 514, 1962.

26. Howard-Pecbles, P.N., Finley, W.II.: Screening of mentaly retarded males for masro orchidism and fragile X chromosome. Am. J. Genct. I5: 631, 1983.

27. Garido, P, Navarete, A. Curotto, B., Alliende, M.A., Zawala, A., Lacassie, Y.: listudio diasnústico genético en pacientes con retraso mental institucionalizados: III. Sindrome de retraso mentil más macroorquidismo sin fragilidad X.. X Jornadas Nacionales de Pediarí, Viña del Mar, Dibiembre 1983.

28. Valenzuele, C. Avendaño, A.: Antropometría y maduración sexual de escolares de un área de Santiago de Chile. Bol. Of. Sanit. Panam. 87: 113 . 1979.

29. De Arce, M.A., Keeris, A.: The iragile X syndrome: the patients and their chromosomes. J. Med. Genet. $21: 84.1984$.

30. Cortada, $X$, Kousseff, R.G.: Cryptorchidism in mental retardation. J. Ural 131: 674, 1984. 